\title{
An efficient system for intestinal on-site butyrate production using novel microbiome-derived esterases
}

\author{
Dah Hyun Jung ${ }^{1,2}$, Ji Hyun Yong ${ }^{1,2}$, Wontae Hwang ${ }^{1,2}$, Mi Young Yoon ${ }^{1,3}$ and Sang Sun Yoon ${ }^{1,2,3,4^{*}}$
}

\begin{abstract}
Short-chain fatty acids, especially butyrate, play beneficial roles in sustaining gastrointestinal health. However, due to limitations associated with direct consumption of butyrate, there has been interest in using prodrugs of butyrate. Tributyrin (TB), a triglyceride composed of three butyrate molecules and a glycerol, is a well-studied precursor of butyrate. We screened a metagenome library consisting of 5760 bacterial artificial chromosome clones, with DNA inserts originating from mouse microbiomes, and identified two clones that efficiently hydrolyse TB into butyrate. Nucleotide sequence analysis indicated that inserts in these two clones are derived from unknown microbes. BLASTp analysis, however, revealed that each insert contains a gene homologous to acetylesterase or esterase genes, from Clostridium spp. and Bacteroides spp., respectively. Predicted structures of these two proteins both contain serine-histidine-aspartate catalytic triad, highly conserved in the family of esterases. Escherichia coli host expressing each of the two candidate genes invariably produced greater amounts of butyrate in the presence of TB. Importantly, administration of TB together with cloned E. coli cells alleviated inflammatory symptoms in a mouse model of acute colitis. Based on these results, we established an efficient on-site and real-time butyrate production system that releases butyrate in a controlled manner inside the intestine.
\end{abstract}

Keywords: Tributyrin, Butyrate, Esterase, Prodrug, Microbiome, Metagenomic library, Colitis, Inflammatory bowel disease (IBD), Bacteroidales, Clostridiales

\section{Introduction}

Our immanent symbiont, the intestinal microbiome, has been intensely studied due to its realised impact on human physiology. Dysbiosis of commensal bacteria and alteration of commensal-derived metabolites in the gut trigger greater vulnerability to pathogenic infections, inappropriate immune response or even systematic disorders [1]. Among various bacterial metabolites produced in the gut, short chain fatty acids (SCFAs) are mainly produced by anaerobic fermentation of indigestible

\footnotetext{
* Correspondence: sangsun_yoon@yuhs.ac

'Department of Microbiology and Immunology, Yonsei University College of Medicine, 50-1 Yonsei-ro, Seodaemun-gu, Seoul 03722, South Korea

${ }^{2}$ Brain Korea 21 PLUS Project for Medical Sciences, Yonsei University College of Medicine, Seoul, Republic of Korea

Full list of author information is available at the end of the article
}

polysaccharides by commensal anaerobes, and SCFAs have been highlighted as a key factor for sustaining gut homeostasis [2]. In particular, butyrate, one of the major SCFAs in the gastrointestinal tract, serves as a major energy source for colonocytes and also a signalling molecule as histone deacetylase inhibitor and a ligand of GPR41 or GPR43 receptor [3, 4]. Ultimately, butyrate can enhance the gut barrier function of intestinal epithelial cells, exert anti-inflammatory effects, and suppress the occurrence of colorectal cancer [5-7].

Inflammatory bowel disease (IBD), of which major subtypes are ulcerative colitis (UC) and Crohn's disease $(\mathrm{CD})$, is a chronic and relapsing inflammatory condition in the gastrointestinal tract accompanied by persistent diarrhoea, abdominal pain, rectal bleeding, and weight 
loss [8]. Though the exact trigger of IBD remains unclear, many research indicate that IBD is a result of inappropriate immune response in genetically susceptible individuals [9].

Emerging evidence strongly suggests that dysbiosis of the gut microbiome and IBD are highly correlated, demonstrated by the compositional differences between the stool samples of IBD patients and healthy controls [10]. A significant change of microbial diversity in the IBD stool samples is decreased abundance of Ruminococcaceae (Clostridium cluster IV) and Lachnospiraceae (Clostridium cluster XIVa) $[10,11]$, which are famous butyrateproducing bacteria [12], compared to stool samples from healthy individuals. Depletion of butyrate in IBD patients might be related to the decreased abundance of butyrateproducing bacteria [13]. Thus, administering either the butyrate-producing bacteria or butyrate itself has been considered as a potential therapeutic agent for the treatment of IBD via its anti-inflammatory effects [14-16].

In spite of the prominent therapeutic potential of butyrate, its actual usage in the clinical setting has been met with scepticism due to the short half-life of butyrate. For instance, when butyrate was intravenously administered to a child with leukaemia $(500 \mathrm{mg} / \mathrm{kg}$ body weight per day) over a period of several days, limited change in prognosis was made, on the account of the short half-life of butyrate of $6 \mathrm{~min}$, and low peak serum levels of butyrate observed [17]. Similarly, a separate study measured the blood concentrations of butyrate, and showed a rapid elimination following the intravenous injection of arginine butyrate [18]. Moreover, clinically utilizing dietary fibre as major source of butyrate also seems infeasible. The estimated amount of dietary fibre required to observe clinical efficacy for an average individual is $136 \mathrm{~g}$ per $1000 \mathrm{kcal}$, which is approximately 9.7 times more than that of the recommended intake of $14 \mathrm{~g}$ per $1000 \mathrm{kcal}$ [19].

In order to overcome this issue of butyrate administration, there has been growing interest in utilizing butyrate derivatives or prodrugs which are more stable and thus more of the administered drug reaches the target site. One of the most prominent prodrugs of butyrate is tributyrin (TB), which is a triglyceride composed of three butyric acid molecules and a glycerol. Butter is one of the richest dietary sources of TB, containing up to $3-4 \%$ of its weight [20]. When TB is ingested, for example through consumption of butter, it gets degraded by hepatic lipases releasing 3 butyrate molecules. Thus, dietary intake of TB is expected to yield similar beneficial results as direct consumption of butyrate. A pharmacokinetic study of TB shows that the plasma half-life of butyrate after TB administration is $40 \mathrm{~min}$, which is longer than that of direct butyrate administration [20]. Furthermore, many studies have shown that TB reduces the level of pro-inflammatory cytokines, induces apoptosis of colonic cancer cells similarly to butyrate [21]. In vivo experiment using a rat model of colon carcinogenesis also showed that TB exerts anti-cancer effects [22].

We hypothesized that gut commensal microbes may play a role in TB metabolism and that the resulting metabolites, especially butyrate, may provide health benefits to host. In this research, we screened a metagenomic library constructed using the mouse gut commensal microbiome and identified two novel esterase genes whose products efficiently breakdown TB into butyrate. Finally, we tested the anti-inflammatory effects of the combination of $\mathrm{TB}$ and commensal Escherichia coli clones that express the esterase genes in dextran sulphate sodium (DSS)-induced colitis mouse model.

\section{Results}

\section{Tributyrin (TB) degrading clones exhibited butyrate producing capability}

Since TB is an ester derived from a glycerol and 3 molecules of butyrate, we postulated that enzymes that can catabolize TB may produce butyrate. Hence, we sought to establish an efficient screening method for high-throughput identification of bacterial clones that express such TBdegrading enzymes. We took advantage of the fact that agar plates containing dissolved TB (Tributyrin Agar, TBA) appear turbid, and that upon TB degradation, the agar turns transparent. Indeed, we observed that bacterial clones producing TB-degrading enzymes, such as PAO1 strain of Pseudomonas aeruginosa, form colonies with halos of a cleared zone on TBA (data not shown). Using this simple yet effective screening scheme (Fig. 1a), we screened a metagenomic library consisting of 5760 bacterial artificial chromosome (BAC) clones, where each DNA insert was purified from the gut microbiome of BALB/c mice [23]. An E. coli $\mathrm{DH} 10 \mathrm{~B}$ that contains the pIndigoBAC-5 vector with no insert was used as negative control. Two clones (33E2 and 54E5) exhibited prominent halos around the colony on TBA following $48 \mathrm{~h}$ growth (Fig. 1b). These two TBdegrading clones were subsequently isolated and evaluated for butyrate production. Quantitative measurement of butyrate using HPLC implicated that 33E2 and 54E5 produced $>8$ times and $>3$ times more butyrate, respectively, than the control strain in the presence of $20 \mathrm{mM}$ of the substrate TB (Fig. 1C). This quantitative butyrate measurement is consistent with what was observed on the TB plate (Fig. 1b). In addition, we also confirmed that overnight cultures of 33E2 and 54E5 produced the characteristic smell which signalled butyrate production.

\section{Sequence analysis of 33E2 and 54E5 reveals the genes responsible for TB degradation}

In order to better understand the genes related to TB degradation, the vectors of 33E2 and 54E5 were 


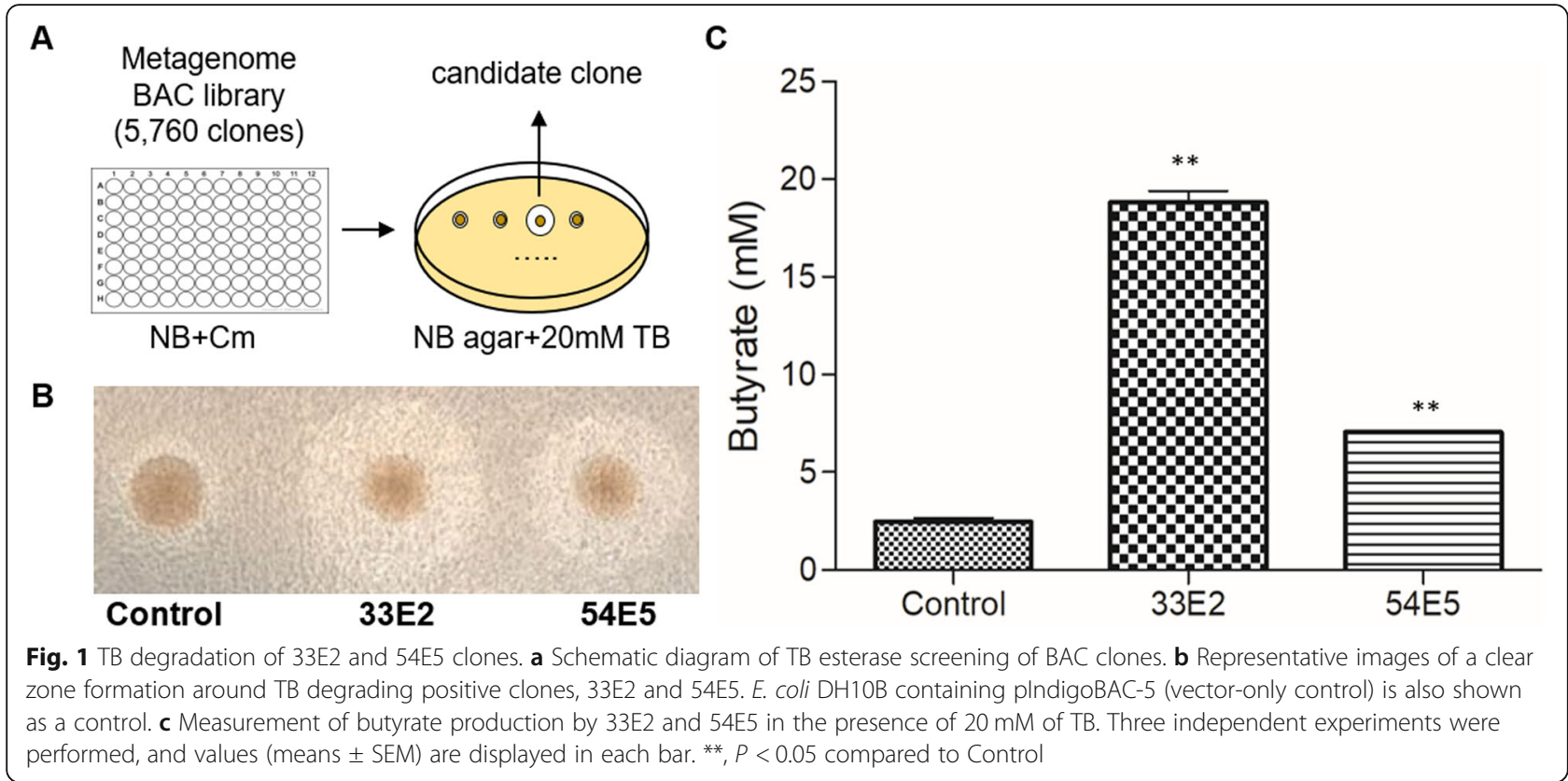

sequenced and analysed. Consequently, the sequence of 33E2, which contains a $10.4 \mathrm{~kb}$ insert, was fully assembled as a complete circular contig. In contrast, the inserted sequence of the 54E5 clone was assembled as three contigs. Based on the lengths of these three contigs, the insert size of the 54E5 clone is estimated to be larger than $64.7 \mathrm{~kb}$.

When BLASTn searches were performed using the sequences of the 33E2 and 54E5 inserts as query, no significant alignment was retrieved, suggesting that the inserts of these two clones are derived from microbes with unknown genome sequences (Tables 1 and 2). However, the 33E2 insert is most similar to a region of Blautia producta genome with $74.3 \%$ identity (Table 1 ). Third, fourth and fifth highest-ranking hits of the BLASTn search were identified as genomic sequences from Clostridium lentocellum, Herbinix sp. and Cellulosilyticum sp. bacteria, respectively, all of which belong to the Lachnospiraceae family $[24,25]$. Contig1 and contig3 of the 54E5 insert share regions of high homology with genomic regions of Duncaniella sp. (Table 2). No sequence with significant homology to contig2 was detected by BLASTn. Disregarding contig2, most of the hits in Table 2 are associated with members of the Muribaculaceae family [26]. These results suggest that the DNA inserts of 33E2 and 54E5 have originated from bacteria belonging to Lachnospiraceae and Muribaculaceae, respectively. Of note, the predicted origin information of the 33E2 and 54E5 DNA fragments are consistent with the information derived from the BLASTp search of amino acid sequences as query.

The sequence of the 33E2 insert contains 9 ORFs including the first ORF that encodes a putative esterase (Fig. 2a). Although the species origin of the DNA fragment cannot be ascertained, all of the ORFs within the 33E2 insert encode proteins highly homologous to those of Lachnospiraceae family, whose members are wellknown producers of short-chain fatty acids [10]. The list of proteins encoded by 33E2 insert genes is provided in Supplementary Information (Table S1). The protein

Table 1 List of BLASTn search results of inserted DNA sequence of 33E2

\begin{tabular}{|c|c|c|c|c|c|c|}
\hline Contig & Rank $^{*}$ & Description & Query Cover & E value & Identity (\%) & Accession \\
\hline \multirow[t]{5}{*}{$33 \mathrm{E} 2$} & 1 & Blautia producta strain PMF1 chromosome, complete genome & $56 \%$ & 0 & 74.3 & CP035945.1 \\
\hline & 2 & Paenibacillus riograndensis SBR5 genome assembly SBR5(T), chromosome: I & $33 \%$ & $1.00 \mathrm{E}-80$ & 63.2 & LN831776.1 \\
\hline & 3 & Clostridium lentocellum DSM 5427, complete genome & $5 \%$ & $2.00 E-34$ & 67.41 & CP002582.1 \\
\hline & 4 & Herbinix sp. SD1D genome assembly SD1D, chromosome: I & $4 \%$ & $2.00 \mathrm{E}-28$ & 67.9 & LN879430.1 \\
\hline & 5 & Cellulosilyticum sp. WCF-2 chromosome, complete genome & $5 \%$ & $6.00 \mathrm{E}-28$ & 66.91 & СР034675.1 \\
\hline
\end{tabular}

* Rank of the result is arranged in order of $E$-value 
Table 2 List of BLASTn search results of inserted DNA sequence of 54E5

\begin{tabular}{|c|c|c|c|c|c|c|}
\hline Contig & Rank $^{*}$ & Description & Query Cover & E value & Identity (\%) & Accession \\
\hline \multirow{5}{*}{$\begin{array}{l}54 \mathrm{E} 5 \\
\text { Contig1 }\end{array}$} & 1 & Duncaniella sp. B8 chromosome, complete genome & $9 \%$ & 0 & 95.53 & СР040121.1 \\
\hline & 2 & Duncaniella sp. C9 chromosome & $9 \%$ & 0 & 95.53 & CP039547.1 \\
\hline & 3 & $\begin{array}{l}\text { Muribaculaceae bacterium DSM } 108610 \text { strain Oil-RF-744-WCA-WT-10 } \\
\text { chromosome, complete genome }\end{array}$ & $9 \%$ & $5.00 \mathrm{E}-133$ & 70.62 & СР045696.1 \\
\hline & 4 & Muribaculum intestinale strain YL27 chromosome, complete genome & $9 \%$ & $9.00 \mathrm{E}-143$ & 87.47 & CP015402.2 \\
\hline & 5 & Muribaculum intestinale strain YL27 genome & $9 \%$ & $9.00 \mathrm{E}-143$ & 87.47 & CP021421.1 \\
\hline \multirow[t]{5}{*}{$\begin{array}{l}54 \mathrm{E} 5 \\
\text { Contig2 }\end{array}$} & 1 & $\begin{array}{l}\text { Uncultured bacterium clone NOD_dss_A3_G08 } 165 \text { ribosomal RNA gene, } \\
\text { partial sequence }\end{array}$ & $5 \%$ & 0 & 99.93 & JQ083840.1 \\
\hline & 2 & $\begin{array}{l}\text { Uncultured bacterium clone WT_ctrl_D1_G10 } 165 \text { ribosomal RNA gene, } \\
\text { partial sequence }\end{array}$ & $5 \%$ & 0 & 99.87 & JQ084982.1 \\
\hline & 3 & $\begin{array}{l}\text { Uncultured bacterium clone NOD_ctrl_C1iii_C05 } 165 \text { ribosomal RNA gene, } \\
\text { partial sequence }\end{array}$ & $5 \%$ & 0 & 99.87 & JQ084661.1 \\
\hline & 4 & $\begin{array}{l}\text { Uncultured bacterium clone WT_dss_B5_H07 16S ribosomal RNA gene, } \\
\text { partial sequence }\end{array}$ & $5 \%$ & 0 & 99.87 & JQ084456.1 \\
\hline & 5 & $\begin{array}{l}\text { Uncultured bacterium clone WT_dss_B1_C01 } 165 \text { ribosomal RNA gene, } \\
\text { partial sequence }\end{array}$ & $5 \%$ & 0 & 99.87 & JQ084160.1 \\
\hline \multirow{5}{*}{$\begin{array}{l}54 \mathrm{E} 5 \\
\text { Contig3 }\end{array}$} & 1 & Duncaniella sp. B8 chromosome, complete genome & $34 \%$ & 0 & 94.98 & СР040121.1 \\
\hline & 2 & Duncaniella sp. C9 chromosome & $34 \%$ & 0 & 94.98 & СР039547.1 \\
\hline & 3 & Duncaniella dubosii strain H5 chromosome & $33 \%$ & 0 & 89.59 & СР039396. \\
\hline & 4 & Uncultured bacterium BAC25G1 genomic sequence & $26 \%$ & 0 & 95.45 & KC595277.1 \\
\hline & 5 & Muribaculum intestinale strain YL27 chromosome, complete genome & $34 \%$ & 0 & 84.81 & CP015402.2 \\
\hline
\end{tabular}

${ }^{*}$ Rank of the result is arranged in order of $E$-value

encoded by ORF1 (795 bp, 240 aa) is most homologous to the alpha/beta hydrolase fold domain-containing protein of Cellulosilyticum lentocellum with $60.46 \%$ amino acid sequence identity. Second-ranked is a protein with the same description and produced by another species of the same genus, Cellulosilyticum (Fig. 2a). Other proteins in the list are acetylesterases, either from the genus of Clostridium or Herbinix (Fig. 2a). These results strongly suggest that TB-degrading capability of the 33E2 clone can be attributed to the enzymatic activity of this gene product.

The sequence of the 54E5 insert contains 32 ORFs and 90.6\% (29 ORFs) of the encoded proteins are highly homologous to proteins from the Bacteroidales order (Fig. 2b). The very first gene in contig3 (1194bp, 397aa) was determined to encode a protein, whose amino acid sequence is highly homologous to a Muribaculaceae bacterium esterase with $86.9 \%$ identity. Proteins retrieved from the BLASTp search using this protein as query similarly include esterases or alpha/beta hydrolases from species of the Bacteroides genus (Fig. 2b). Hereafter, we named ORF1 of 33E2 and ORF1 of the 54E5 contig1 as tbe 1 and tbe2, respectively, with tbe standing for "tributyrin esterase".

\section{Characterization of Tbe 1 and Tbe2}

In an effort to shed light on the molecular nature of Tbe1 and Tbe2, the respective amino acid sequences were examined using InterPro and SignalP5.0. Analysis of Tbe1 revealed the lack of a signal peptide in the amino acid sequence implying that Tbe1 is probably an intracellular protein. In contrast, Tbe 2 amino acid sequence includes a signal peptide sequence, from residue 1 to 19 (Fig. 3a, red underlined), which suggests that Tbe2 may be an extracellular protein. Furthermore, amino acid sequences of Tbe1 and Tbe 2 were compared with those of the known TB esterases of Lactobacillus lactis (LL_Tbe) and Streptococcus pneumoniae (SP_Est) [27, 28]. All of the proteins contained an alpha-beta hydrolase conserved domain (InterPro entry: IPR029058), which is common to hydrolytic enzymes (Fig. 3a). In addition, we also detected the presence of a serine-histidine-aspartate (SHD) catalytic motif, included within the active sites of most alpha-beta hydrolase fold super family proteins [29] (Fig. 3a). Furthermore, all SHD motifs found in our analysis contained 3 consensus sequences (LLHG, GLSMGG, and DFL) (Fig. 3a, highlighted in black).

We then asked whether Tbe1 and Tbe 2 proteins are structurally similar to a known esterase. Structures of Tbe 1 and Tbe 2 were simulated based on the 3D structure of S. pneumoniae EstA [28] from the RSCB Protein Data Bank (PDB entry: 2UZ0) as template. Tbe1 shares the overall structural similarity with the template to a greater extent than Tbe2 does (Fig. 3b). However, the SHD triad, found in both Tbe1 and Tbe2 conserved within the catalytic core region, is nicely superimposed with that of the $S$. pneumoniae esterase. These results strongly suggest that Tbe1 and Tbe 2 are responsible for 


\begin{tabular}{|c|c|c|c|c|}
\hline \multicolumn{5}{|c|}{ A $33 \mathrm{E} 2$} \\
\hline T & 1 & T & T & T \\
\hline Rank & Description & Total score & Query cover $(\%)$ & Identity $(\%)$ \\
\hline 1 & alpha/beta hydrolase fold domain-containing protein [Cellulosilyticum lentocellum] & 353 & 99 & 60.46 \\
\hline 2 & alpha/beta hydrolase fold domain-containing protein [Cellulosilyticum sp. WCF-2] & 350 & 99 & 60.08 \\
\hline 3 & acetylesterase [Clostridium sp. E02] & 336 & 99 & 58.56 \\
\hline 4 & acetylesterase [Herbinix hemicellulosilytica] & 341 & 99 & 58.33 \\
\hline 5 & acetylesterase [Clostridiales bacterium] & 338 & 99 & 58.33 \\
\hline
\end{tabular}

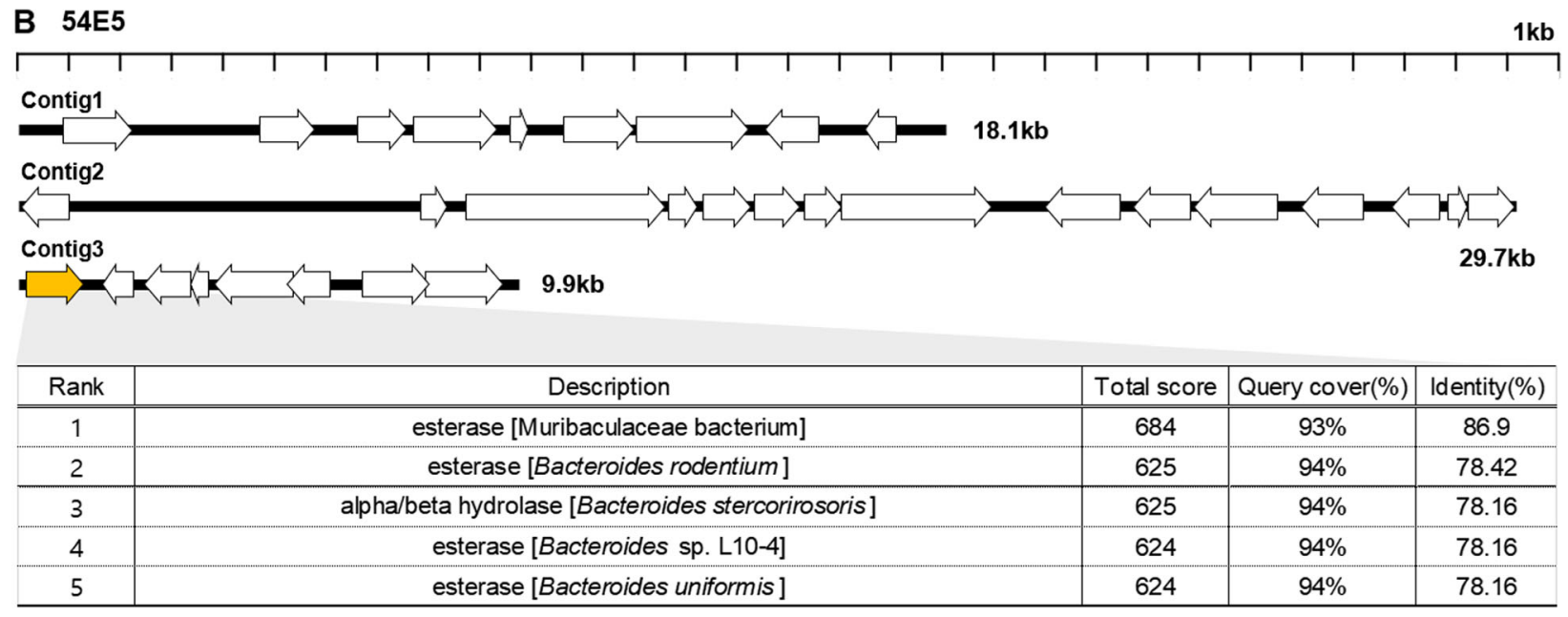

Fig. 2 Open reading frame (ORF) maps and BLASTp results of 33E2 and 54E5 clones. ORF maps of the (a) $33 \mathrm{E} 2$ and (b) 54E5 clones. The length and direction of the arrows indicate the relative size and direction of each ORF. Detailed information of the ORFs is described in supplementary information. ORFs encoding putative esterases, Tbe1 and Tbe2, are coloured red and orange, respectively, and the top 5 BLASTp hits of the ORFs are listed in the table arranged by increasing e-value

TB degradation and butyrate biosynthesis in the 33E2 and 54E5 clones.

\section{Relative activity of Tbe1 or Tbe2 with the known TB esterases}

Next, we compared the enzyme activity of Tbe1 and Tbe 2 with previously reported tributyrin esterases [27, 30]. To this end, we further cloned genes encoding the esterase of Lactococcus lactis (LL_Tbe) and Streptococcus pneumoniae (SP_Est). In order to evaluate the enzyme activities under the same condition, all four genes were cloned into the same location in the pBAD24 plasmid, which is downstream of the promoter sequence of tbe1. The bacterial strains were then incubated with 4nitrophenyl butyrate ( $p$-NPB), a substrate of the enzyme. Hydrolysis of $p$-NPB by the esterase enzyme releases 4nitrophenolate, which can be detected by absorbance at $400 \mathrm{~nm}$. Based on Fig. 4, the esterase of S. pneumoniae was the most potent among the four tested enzymes. Of note, Tbe 2 is more active than Tbe1, and Tbe1 demonstrated almost identical activity to the L. lactis enzyme.
While using purified enzymes is more preferable for a precise quantification of the enzyme's kinetic parameters, these results clearly suggest that Tbe1 and Tbe2, which we identified in the current study, are either as equally as or more active than the esterase of L. lactis, a probiotic strain.

\section{Tbe1 and Tbe2 degrade TB and produce butyrate}

Our bioinformatic analyses clearly suggest that Tbe 1 and Tbe 2 are the most probable proteins contributing to conversion of TB into butyrate. In order to conclusively determine the roles of Tbe1 and Tbe2, we cloned tbe1 and tbe 2 genes into another E. coli host and monitored TB-induced butyrate production. The host strain we used for Tbe expression is the $t E c$ strain that we isolated from the mouse intestines [31]. tbe1 gene was amplified to include $\sim 100 \mathrm{bp}$ sequence upstream of the tbe1 ORF and cloned into pBAD24 plasmid (pBAD24::tbe1). Inclusion of the upstream sequence was intended to enable transcription of tbe 1 using its endogenous promoter. tbe 2 gene was inserted right at the junction of the 54E5 


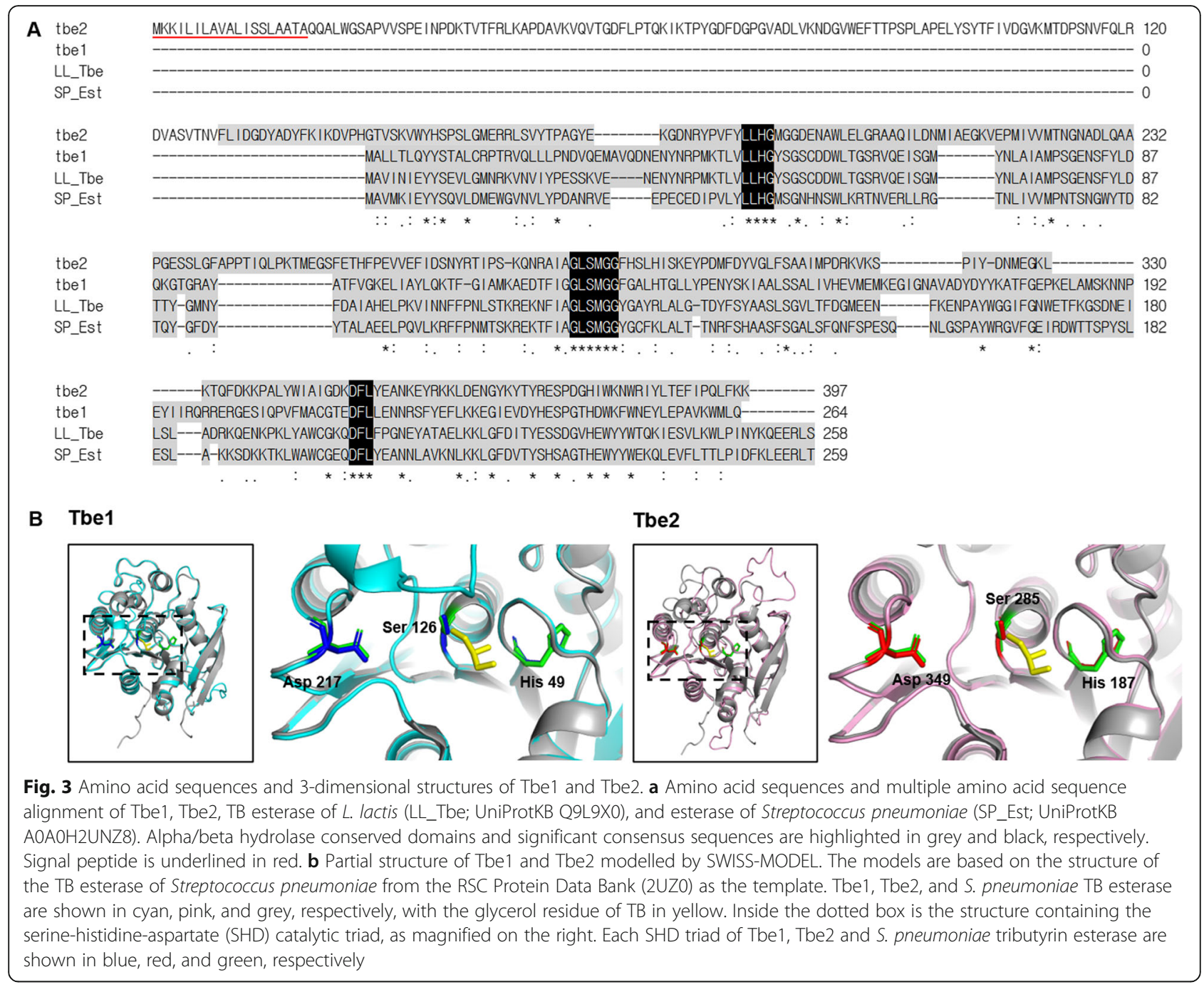

plasmid (Fig. 2b), so we were unable to clone tbe 2 with its endogenous promoter. Therefore, tbe 2 gene was cloned in place of the tbe1 ORF in pBAD24::tbe1, and the resultant plasmid was named pBAD24::tbe2. When $t E c$ strains harbouring either plasmids were grown in the presence of $5 \mathrm{mMTB}$, prominent increases in butyrate production level were observed (Fig. 5). Butyrate production levels in these two cultures were $>16$ times higher than the control culture. The characteristic scent of butyrate was again noticed in these two cultures.

Of interest, the level of butyrate produced by tbe 2 gene was almost identical to that by tbe1 gene. In Fig. 1c, butyrate production by 54E5 was substantially less than that by 33E2. We speculate that this discrepancy was caused by the absence of tbe 2 gene's own endogenous promoter in the original 54E5 clone. In 54E5, tbe 2 gene expression was probably induced by the upstream sequence part of the pIndigoBAC-5 plasmid. When $t b e 2$ was transcribed in the presence of the $t b e 1$ promoter in pBAD24::tbe2, greater butyrate production was achieved.
In vivo on-site butyrate production protects mice against DSS-induced colitis

Our results so far demonstrate that TB together with Tbe-expressing E. coli cells can efficiently produce butyrate, a beneficial microbiome-derived metabolite. To examine whether or not butyrate produced by this system can alleviate intestinal inflammation, we used DSSinduced mouse model of acute colitis. Mice were divided into 4 groups that received different treatments, as illustrated in Fig. 6a. Mice administered 2.5\% DSS developed acute colitis characterized by weight loss, bloody diarrhoea, and watery stool, and these outcomes were collectively reflected in the increase of the DAI (Disease Activity Index) score [32] (Fig. 6b). In TB-only group, the DAI scores remained persistently high even after DSS administration was discontinued, suggesting that TB itself did not exert any positive effects toward restoration of a healthy gut condition in mice. It was of particular interest that $E$. coli cells expressing tbe 2 provided significant beneficial effects in mice suffering from 


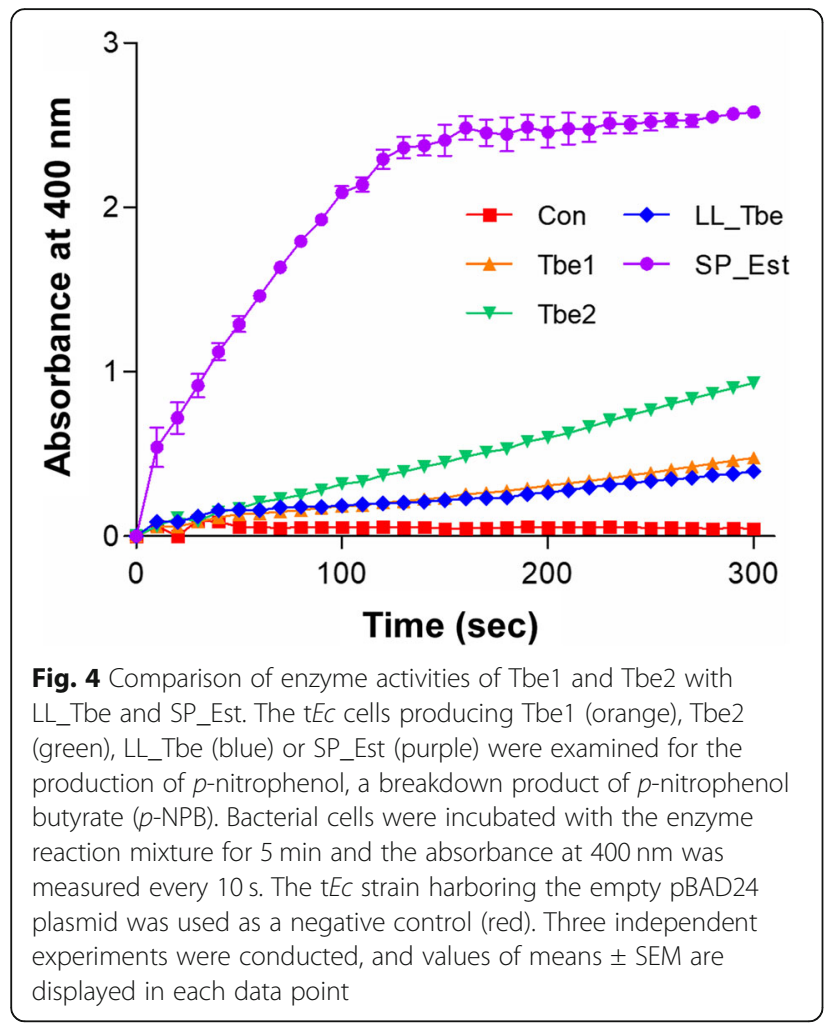

severe colitis (Fig. 6b). E. coli cells expressing tbe1 did not seem to confer any protective effects in comparison to the empty vector control group (Fig. 6b). It is not clear why only tbe2-expressing $E$. coli cells led to the amelioration of DAI scores.

Another phenotype of the DSS-induced acute colitis is the shortened intestinal length as gut inflammation

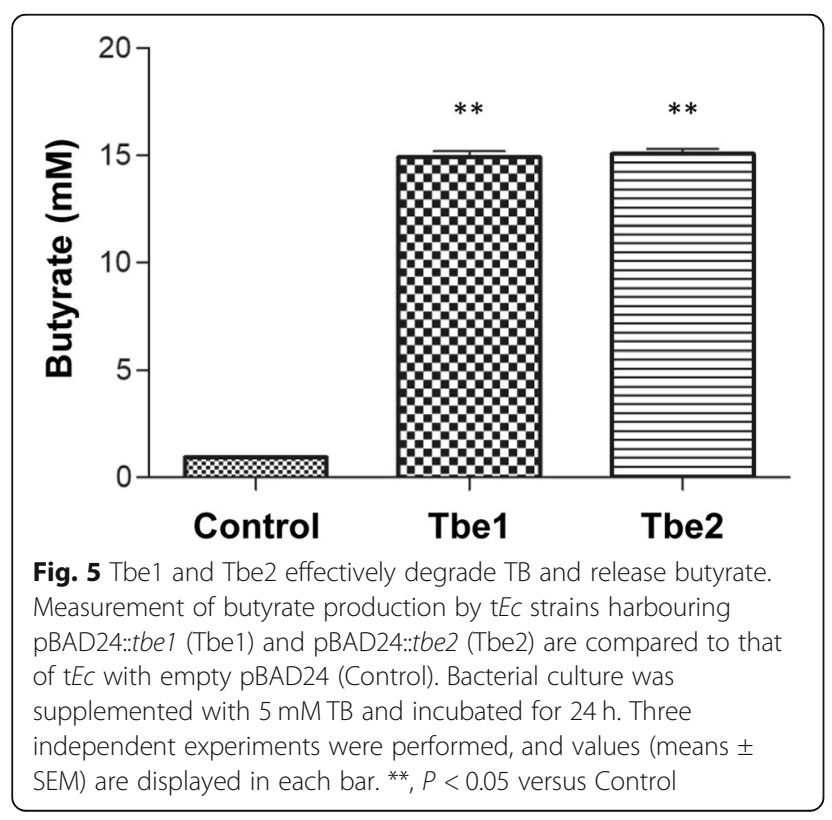

manifests [33]. On day 12, the last day of the experiment, mice from each group were sacrificed and the lengths of their colons were measured. The colon lengths of mice that received Tbe1- or Tbe2-expressing $E$. coli cells were significantly longer than those of control mice (Fig. 6c), indicating the positive effects of Tbe against DSS-induced colitis. Moreover, the intestinal tissues looked significantly improved by Tbe1 or Tbe2. Hyper-inflammatory responses evidenced by neutrophil infiltration (black arrow) and fluid accumulation (white arrows) were observed in DSS-pretreated mice that received only TB (Fig. 6d). Still higher degrees of neutrophil staining were observed in mice that received control E. coli cells (Fig. 6e, black arrow). In contrast, intestinal tissues looked considerably improved when DSSpretreated mice were treated with $\mathrm{TB}$ and $E$. coli cells that express Tbe1 (Fig. 6f) or Tbe2 (Fig. 6g). These results clearly suggest that on-site butyrate production by coupling TB and microbiome-derived novel esterases can exert beneficial effects in the inflamed mouse intestines.

\section{Discussion}

Though computational analysis has enabled significant progress to be made in recent years, functional metagenomic studies using conventional screening methods still remain irreplaceable and invaluable in the identification of novel genes and characterization of their functions. Metagenomic libraries make it feasible to elucidate the functions of genes from unculturable microbes, which has remained a major challenge in gut microbiome studies. For instance, several niche adaptive features of the gut microbiome such as carbohydrate utilization, colonization factors, bile acid resistance and antibiotic resistance have been revealed through the use of metagenomic libraries constructed from the gut commensal microbiomes of either human or other mammals [34-37].

Since dietary fibres are a major source for SCFAs in our gut, many gut metagenome studies have focused on identifying the genes responsible for the metabolism of dietary fibres [38]. Due to the differences in microbiome compositions among individuals, the level of SCFAs produced from dietary fibres varies significantly [39]. Therefore, it would be of crucial benefit to strategize an efficient system of delivering SCFAs in a controlled manner. Here, we established an on-site butyrate production system (OBPS) using a butyrate precursor and microbiome-derived esterases. We chose TB as a prodrug of butyrate and established a functional metagenome screening method that enabled the identification of genes encoding enzymes that specifically catalyse the conversion of TB to butyrate.

The success of our screening method is attributed to the following elements. Firstly, using Triton X-100, we 

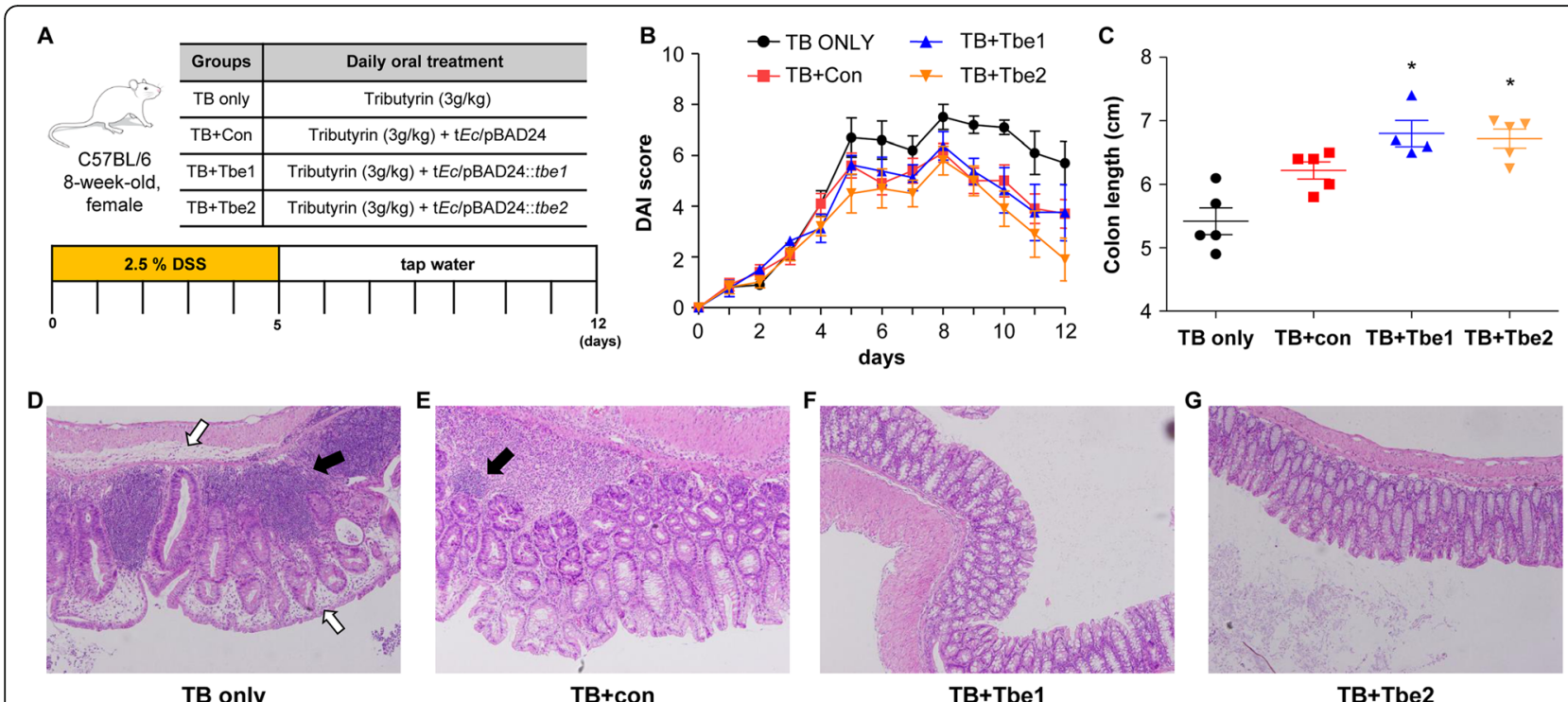

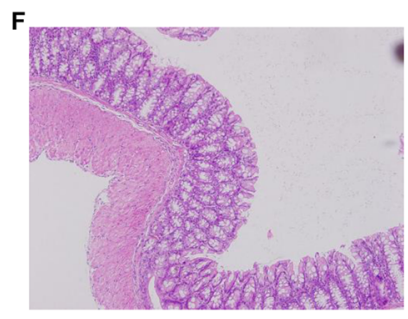

TB+Tbe1

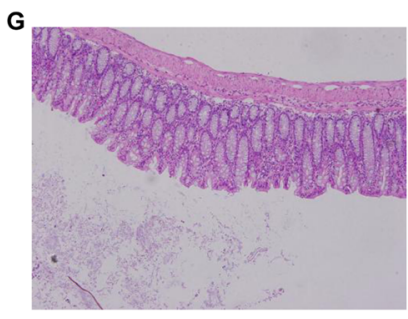

TB+Tbe2

Fig. 6 Effects of TB and Tbe on dextran sulphate sodium (DSS)-induced colitis. a Schematic diagram of DSS-induced colitis model, $\mathbf{b}$ disease activity index (DAI) score, and c colon length of colitis induced mice treated with only TB (TB ONLY), TB with tEc with empty pBAD24 (TB + Con), tEc with pBAD24::tbel ( $T B+T b e 1)$ and tEc with pBAD24::tbe2 (TB + Tbe2). (D-G) Representative images of H\&E stained sections of colons from indicated groups. Arrows denote neutrophil infiltration (black) and edema (white). ${ }^{*}, P<0.05$ versus TB + Con

were able to homogenously solidify agar plates that contain highly insoluble TB. TB agar plates acted as an incredibly useful platform, via which high-throughput screening of a large genomic library was performed with minimal plate-to-plate variations. Secondly, the characteristic scent of butyrate aided the differentiation of positive clones from negative ones, especially during the verification stage. Thirdly, TB is a commonly used substrate in esterase assays, yet previous studies have mostly focused on the enzymes rather than the products of the enzymatic reactions. We focused on the fact that TB is an ester of 3 molecules of butyrate condensed with glycerol, and that positive clones would likely generate butyrate as the major product of TB degradation. Fourthly, TB, as a food-grade lipid, can safely be fed to live animals as a prodrug of butyrate, with no expected side-effects. Thus, TB could be safely administered to experimental animals and enabled in vivo confirmation of beneficial effects of TB combined with esterase containing clones.

Until now, TB esterase has gained attention in the context of fermented food and agricultural industries $[27,40,41]$. Here, we report the discovery of novel esterases in the gut, Tbe1 and Tbe2. These esterases were predicted to have originated from two separate phyla; Firmicutes and Bacteroidetes, two predominant phyla of the human intestine [42]. Given that these phyla are highly abundant and commonly found across individuals irrespective of geography, race, gender and other characteristics, we speculate that this esterase function serves a physiologically significant role and is inherent across a broad spectrum of microbial species in the intestine.
Interestingly, Tbe 1 and Tbe 2 share a common catalytic feature. Alpha/beta hydrolase domain is highly conserved among various hydrolytic enzymes [43]. Serine residue of the Ser-His-Asp (SHD) catalytic triad, a typical catalytic motif found in the alpha/beta hydrolase domain, is an essential residue for the enzymatic activity of esterases [30] and often included in the consensus sequence (Gly-x-Ser-x-Gly; $x$ refers to any amino acid) [29]. The amino acid sequences of Tbe1 and Tbe2 both contain the sequence GLSMGG which is found in other known TB esterases. The other two residues, histidine and aspartate, are included in the other two consensus sequences, LLHG and DFL, respectively.

The DNA fragment insert of 33E2 is highly homologous to the genome of Lachnospiraceae. Lachnospiraceae is a gram-positive family that belongs to the Firmicutes phylum. Genera such as Blautia, Coprococcus, Dorea, Lachnospira, Oribacterium, and Roseburia are included in this family. Members of Lachnospiraceae are specialised in utilising plant derived-polysaccharides such as starch, inulin, and arabinoxylan [44]. Similar to Bacteroides spp. which have well-established carbohydrate utilization systems encoded within polysaccharide utilization loci (PUL), several members of Lachnospiraceae have been reported to possess similar genomic features termed gram-positive PUL (gpPUL). gpPUL is defined as a collection of genes encoding at least one of 3 components: polysaccharidedegrading enzyme, a carbohydrate transport system and a transcriptional regulator [44]. Moreover, esterases are frequently present within a gpPUL. Some ORFs of 33E2 correspond with the 3 components of gpPUL; ORF7 and 
ORF8 are predicted as $A B C$ transporter permeases, and ORF9 is a response regulator. Hence, it seems highly possible that Tbe1 of 33E2 is involved in carbohydrate degradation of Lachnospiraceae. The carbohydrate degrading ability of Tbe1 may not be limited to TB, but also be extended to include other various polysaccharides, particularly those that are indigestible by host.

In addition, we examined the effects of TB esterase and TB oral supplementation on the gut barrier function in DSS-induced colitis mouse model. TB delivered via the oral route can be hydrolysed by pancreatic and gastric lipases. Hence, many studies have demonstrated that solely administered TB mediates an anti-inflammatory effect. Mounting evidence suggest that supplementation of TB could induce desirable phenotypes in diet-induced obesity model [45], in piglets [46, 47], and in a murine model of Clostridium difficile infection [48]. In this study, we identified microbiome-derived genes encoding active TB esterases and sub-cloned them into an E. coli strain with strong intestinal colonization capability [31]. When those strains were delivered together with TB into the mouse intestines, on-site production of butyrate was achieved. More importantly, thus produced butyrate was sufficient for alleviating the inflammatory symptoms in the mouse model of acute colitis.

In the present study, we report the finding of two novel TB esterases, yet our experiments have the following limitations: (i) the limited amount of information regarding $\mathrm{TB}$ esterases present in the gut meant that there was no suitable esterase to compare TB degrading activities of the enzymes to; (ii) TB was the only substrate tested in this study and the substrate specificity of Tbe1 and Tbe 2 has not yet been fully explored; (iii) given that Tbe1 likely originated from gram-positive Lachnospiraceae, E. coli might not be an appropriate surrogate host for Tbe1 expression; (iv) despite strenuous efforts, we were unable to detect consistently elevated levels of butyrate in mouse faecal matters discharged from groups of $\mathrm{TB}+\mathrm{Tbe} 1$ or $\mathrm{TB}+\mathrm{Tbe} 2$. We expect that this can be attributed to the rapid metabolism of butyrate by host colonocytes. Further investigations of real-time butyrate production in mouse intestines are required to better rationalize the therapeutic use of OBPS.

Commercially available probiotics are often limited in that the delivered bacterial strains have difficulty stably colonizing the recipient gut and that sufficient amounts of dietary fibre must be available for bacterial cells to exert beneficial effects. However, the OBPS is advantageous in that it is simple, yet the level of butyrate produced can be controlled by the administrator. Therefore, given the abundant evidence for the significance of butyrate in the maintenance of a healthy gut [5-7], the OBPS may prove to be an invaluable treatment option for various diseases.

\section{Materials and methods}

\section{Bacterial strains and culture conditions}

The procedure for BAC (Bacterial Artificial Chromosome) library construction was described in detail elsewhere [23]. Briefly, bacterial DNA was extracted from the combined caecal and colon contents of seven BALB/ c mice and digested with HindIII restriction enzyme. The size-selected DNA was cloned into pIndigoBAC-5 (HindIII cloning-ready; Epicentre), and transformed into E. coli DH10B. Successfully transformed E. coli DH10B was grown in Luria Broth (LB, $10 \mathrm{~g}$ tryptone, $5 \mathrm{~g} \mathrm{NaCl}$, $5 \mathrm{~g}$ yeast extract per litre) supplemented with $25 \mu \mathrm{g} / \mathrm{ml}$ of chloramphenicol (Duchefa Biochemie, Haarlem, Netherlands) at $37^{\circ} \mathrm{C}$. The indigenous non-pathogenic $E$. coli strains $t E c$ (typical $E$. coli) was isolated from $C D-1$ mouse intestines in a previous study [31]. Lactococcus lactis (KCTC 3619) was cultured anaerobically in Brain Heart Infusion (BHI) broth at $37^{\circ} \mathrm{C}$. Streptococcus pneumoniae (ATCC 49619) was cultured aerobically in BHI broth at $37^{\circ} \mathrm{C}$. This information is summarised in Table 3.

\section{Screening of BAC library clones for TB degrading ability}

For TB degradation assay, bacteria were grown on $\mathrm{TB}$ agar (TBA; $4 \mathrm{~g}$ peptone, $3 \mathrm{~g} \mathrm{NaCl}, 3 \mathrm{~g}$ yeast extract, $15 \mathrm{~g}$ agar, $20 \mathrm{mM}$ TB per litre and $0.02 \%$ of triton $\mathrm{X}-100$ as a surfactant) at $37^{\circ} \mathrm{C}$ for $48 \mathrm{~h}$. As TB in the media gets degraded by the hydrolytic activity of bacteria, distinct clear zones form around the bacterial colonies.

\section{Measurement of butyrate}

Bacteria were inoculated into $3 \mathrm{ml}$ of TB broth (TBA without agar) within $50 \mathrm{ml}$ conical tubes and incubated at $37^{\circ} \mathrm{C}$ for $24 \mathrm{~h}$. TB concentrations used for the initial screening of the metagenomic library and for confirmation of successfully transformed $t E c$ clones were $20 \mathrm{mM}$ and $5 \mathrm{mM}$, respectively. Bacterial culture supernatants were filtered using $0.2 \mu \mathrm{m}$ Minis$\operatorname{art}^{\circ}$ Syringe Filters (Sartorius, Germany). Butyrate concentration was measured by high-performance liquid chromatography (HPLC) using Ultimate3000 (Thermo Dionex, USA) with a UV detector $(210 \mathrm{~nm})$ and refractive index detector (RefractoMAX520, Japan). For analysis, the Aminex $87 \mathrm{H}$ column $(300 \times$ $10 \mathrm{~mm}$, Bio-Rad, CA, USA) was used and $0.01 \mathrm{~N}$ $\mathrm{H}_{2} \mathrm{SO}_{4}$ (Fluka, USA) were used as an eluent.

\section{Sequence analysis of the inserted fragments of 33E2 and 54E5}

Whole genome sequencing and de novo assembly of BAC plasmids were performed by Macrogen, Inc. (Seoul, Korea). ORFs were detected using the ORFfinder provided by the National Center of Biotechnology Information (NCBI). Sequence homology searches were 
Table 3 Bacterial strains and plasmids used in this study

\begin{tabular}{|c|c|c|}
\hline Strains and plasmids & Relevant characteristic & Source \\
\hline \multicolumn{3}{|l|}{ E. coli strains } \\
\hline $\mathrm{DH} 10 \mathrm{~B}$ & plndigoBAC-5 without inserted gene & Laboratory collection \\
\hline BAC library & $\mathrm{DH} 10 \mathrm{~B}$, plndigoBAC-5 harboring BALB/C mouse gut microbiome DNA & {$[23]$} \\
\hline $\mathrm{t} E \mathrm{C}$ & wild type strain isolated from CD-1 mouse intestines & [31] \\
\hline tbe1 & $\mathrm{t} E \mathrm{C}, \mathrm{pBAD} 24: \mathrm{tbe} 1$ & This study \\
\hline tbe2 & $\mathrm{tEC}, \mathrm{pBAD} 24:$ tbe2 & This study \\
\hline L. lactis & Type strain KCTC 3619 & Laboratory collection \\
\hline S. pneumoniae & Type strain ATCC 49619 & Laboratory collection \\
\hline \multicolumn{3}{|l|}{ Plasmids } \\
\hline plndigoBAC-5 & $C p^{r}$, library backbone vector & Laboratory collection \\
\hline pBAD24 & Ampr', cloning vector & Laboratory collection \\
\hline
\end{tabular}

performed against the NCBI database using BLASTn and BLASTp algorithms. ORFs were confirmed with Prokka [49], and any candidates without significant hits with BLASTp were excluded from analysis. Conserved domain search was done by using InterPro database and signal peptide sequence of protein was predicted based on SignalP 5.0 [50].

\section{Construction of $E$. coli heterologously expressing tbe 1 and tbe2}

pBAD24 with an ampicillin resistance marker was used to construct the protein expression vectors (Table 3). The DNA fragment of tbe 1 including the endogenous promoter region $(\sim 899 \mathrm{bp})$ was PCR amplified from the 33E2 clone. The DNA fragment of tbe 2 was PCR amplified from 54E5. Since the endogenous promoter region of tbe 2 was not included in the vector of 54E5, the promoter region of tbe1 was amplified and combined with the amplified fragment of tbe 2 by overlapping PCR ( $1298 \mathrm{bp})$. PCR products were cloned into the multicloning site of the pBAD24, and the cloned vectors, named pBAD24::tbe1 and pBAD24::tbe2, were transformed into E. coli DH10B for amplification. Again, the vectors were extracted from $E$. coli DH10B using AccuPrep Plasmid Mini Extraction kit (Bioneer, Korea) and transformed into $t E c$. PCR primers used for cloning are listed in Table 4.

\section{Esterase enzyme activity assay}

Tributyrin esterase activity was assessed following procedures described elsewhere [51], using a chromogenic substrate, $p$-nitrophenyl butyrate ( $p$-NPB, Sigma Aldrich, USA). Production of $p$-nitrophenol was monitored spectrophotometrically by measuring absorbance at $400 \mathrm{~nm}$. For this assay, $t E c$ transformed with the esterase gene was cultivated for $6 \mathrm{~h}$ in LB broth, and then $20 \mu \mathrm{l}$ aliquot was added to the enzyme reaction mixture of final volume $200 \mu \mathrm{l}$.

\section{Dextran sodium sulphate (DSS) mouse model of acute colitis}

Acute colitis was induced in 8-week-old female C57BL/6 mice administered $2.5 \%$ dextran sodium sulphate (DSS; molecular weight, 36,000-50,000 Da; MP Biomedicals, $\mathrm{OH}$, USA) in their drinking water for 5 days, which was replaced with normal drinking water for the following 7 days. The mice were monitored daily for body weight, stool consistency and stool bleeding. Disease activity index (DAI) scores were taken in accordance with a previous study [32]. Over the 7-day period following the DSS administration, mice were orally dosed with TB (3 $\mathrm{g} / \mathrm{kg}$ ) combined with the respective treatments. The treatments delivered were PBS, $\mathrm{t} E c$-pBAD24 (empty vector), $\mathrm{t} E c-\mathrm{pBAD} 24:: t b e 1$, and $\mathrm{t} E c-\mathrm{pBAD} 24:: t b e 2$, respectively. The dosage of each bacterial clones provided in

Table 4 Primers used in this study

\begin{tabular}{|c|c|c|}
\hline Gene name & Direction & Primer sequence $\left(5^{\prime}-3^{\prime}\right)^{\mathrm{a}}$ \\
\hline tbe1_promoter_Xmal & Forward & ACTGCCCGGGAGGTCTCACGGATTCAGGAA \\
\hline tbe1_Xbal & Reverse & ACTCTCTAGATCATTGCAGCATCCATTTAA \\
\hline tbe1_promoter_Xbal & Forward & ACTCTCTAGATTGTAAATAACTCAGATTTGGT \\
\hline tbe1_protmoter_overlapped & Reverse & $\underline{\text { AATTTITTCATATTTCCTCCTGGTATTGCG }}$ \\
\hline tbe2_overlapped & Forward & CAGGAGGAAAATATGAAAAAAATTCTAATCCT \\
\hline tbe2_Pstl & Reverse & ACTCCTGCAGTTATTTCTTGAAAAGTTGAG \\
\hline
\end{tabular}

${ }^{\mathrm{a}}$ Restriction enzyme recognition sequences and overlapped sequences are underlined 
combination with TB were $1 \times 10^{8} \mathrm{CFU}$. The mice were euthanized by $\mathrm{CO}_{2}$ on the 12th day of the experiment and colon lengths were measured.

\section{Histological analysis}

Mouse colons of $3 \mathrm{~mm}$ were harvested, opened longitudinall and fixed in 3.7\% formaldehyde. Tissues were paraffin-embedded and $5 \mu \mathrm{m}$ sections were stained with haematoxylin-eosin (H\&E). Slides were analysed using an Olympus Microscope (Model. U-LH100HG).

\section{Supplementary Information}

The online version contains supplementary material available at https://doi. org/10.1186/s13036-021-00259-4.

Additional file 1: Table S1. Predicted genes in BAC clones.

\section{Abbreviations}

UC: Ulcerative colitis; CD: Crohn's disease; GPR: G-protein-coupled receptor; IBD: Inflammatory bowel disease; SCFA: Short-chain fatty acid; TB: Tributyrin; TBA: Tributyrin agar; BAC: Bacterial artificial chromosome; CFU: Colony forming unit; DSS: Dextran sulfate sodium; Cp: Chloramphenicol; Amp: Ampicillin; Tbe: Tributyrin esterase; SHD triad: Serine-histidine-aspartate triad; OBPS: On-site butyrate production system; PUL: Polysaccharide utilization loci; gpPUL: Gram positive polysaccharide utilization loci; DAl: Disease activity index

\section{Acknowledgements}

The authors would like to thank members of the Yoon lab for critical reading of the manuscript

\section{Authors' contributions}

D.H.J and S.S.Y. conceptualized and designed the experiments. D.H.J, J.H.Y., W.H., M.Y.Y and S.S.Y performed experiments and analyzed experimental results. D.H.J., J.H.Y., and S.S.Y drafted the manuscript. The authors read and approved the final manuscript.

\section{Funding}

This work was supported by grants from the National Research Foundation (NRF) of Korea, which is funded by the Korean Government

(2017M3A9F3041233 and 2019R1A6A1A03032869, 2019R111A1A0105803712). This work was also supported by Korea Institute of Planning and Evaluation for Technology in Food, Agriculture, Forestry and Fisheries (IPET) through Agricultural Microbiome R\&D Program, funded by Ministry of Agriculture, Food and Rural Affairs (MAFRA) (918003041SB010). This research was also supported by a grant of the Korea Health Technology R\&D Project through the Korea Health Industry Development Institute (KHIDI), funded by the Ministry of Health \& Welfare, Republic of Korea (HI14C1324). The authors have declared no conflicts of interest.

\section{Availability of data and materials}

The majority of data generated or analyzed during this study are included in this published article or in the supplementary information. The data not shown in the manuscript are available from the corresponding author on reasonable request.

\section{Ethics approval and consent to participate}

All mouse experiments were conducted according to the guidelines provided by the Department of Animal Resources of Yonsei Biomedical Research Institute. The Committee on the Ethics of Animal Experiments at Yonsei University College of Medicine approved this study (Permit numbers, 2017-0210 and 2018-0250).

\section{Consent for publication}

This manuscript contains no details, or videos relating to an individual person so that this section is not applicable.

\section{Competing interests}

The authors declare that they have no conflicts of interest with the contents of this article.

\section{Author details}

'Department of Microbiology and Immunology, Yonsei University College of Medicine, 50-1 Yonsei-ro, Seodaemun-gu, Seoul 03722, South Korea. ${ }^{2}$ Brain Korea 21 PLUS Project for Medical Sciences, Yonsei University College of Medicine, Seoul, Republic of Korea. ${ }^{3}$ Severance Biomedical Science Institute, Yonsei University College of Medicine, Seoul, Republic of Korea. ${ }^{4}$ Institute for Immunology and Immunological Diseases, Yonsei University College of Medicine, Seoul, Republic of Korea.

Received: 8 November 2020 Accepted: 4 February 2021

Published online: 06 March 2021

\section{References}

1. Maloy KJ, Powrie F. Intestinal homeostasis and its breakdown in inflammatory bowel disease. Nature. 2011;474(7351):298-306.

2. Koh A, De Vadder F, Kovatcheva-Datchary P, Backhed F. From dietary Fiber to host physiology: short-chain fatty acids as key bacterial metabolites. Cell. 2016;165(6):1332-45

3. Johnstone RW. Histone-deacetylase inhibitors: novel drugs for the treatment of cancer. Nat Rev Drug Discov. 2002;1(4):287-99.

4. Chang PV, Hao L, Offermanns S, Medzhitov R. The microbial metabolite butyrate regulates intestinal macrophage function via histone deacetylase inhibition. Proc Natl Acad Sci U S A. 2014;111(6):2247-52.

5. Ma X, Fan PX, Li L, Qiao S, Zhang G, Li D. Butyrate promotes the recovering of intestinal wound healing through its positive effect on the tight junctions. J Anim Sci. 2012;90(suppl_4):266-8.

6. Matter K, Aijaz S, Tsapara A, Balda MS. Mammalian tight junctions in the regulation of epithelial differentiation and proliferation. Curr Opin Cell Biol. 2005:17(5):453-8.

7. Goncalves P, Martel F. Butyrate and colorectal cancer: the role of butyrate transport. Curr Drug Metab. 2013;14(9):994-1008.

8. Baumgart DC, Carding SR. Inflammatory bowel disease: cause and immunobiology. Lancet. 2007;369(9573):1627-40.

9. Zhang YZ, Li YY. Inflammatory bowel disease: pathogenesis. World J Gastroenterol. 2014:20(1):91-9.

10. Halfvarson J, Brislawn CJ, Lamendella R, Vazquez-Baeza Y, Walters WA, Bramer LM, et al. Dynamics of the human gut microbiome in inflammatory bowel disease. Nat Microbiol. 2017;2:17004.

11. Matsuoka K, Kanai T. The gut microbiota and inflammatory bowel disease. Semin Immunopathol. 2015;37(1):47-55.

12. Duncan SH, Barcenilla A, Stewart CS, Pryde SE, Flint HJ. Acetate utilization and butyryl coenzyme a (CoA):acetate-CoA transferase in butyrateproducing bacteria from the human large intestine. Appl Environ Microbiol. 2002;68(10):5186-90

13. Marchesi JR, Holmes E, Khan F, Kochhar S, Scanlan P, Shanahan F, et al. Rapid and noninvasive metabonomic characterization of inflammatory bowel disease. J Proteome Res. 2007;6(2):546-51.

14. Miguel MA, Lee SS, Mamuad LL, Choi YJ, Jeong CD, Son A, et al. Enhancing butyrate production, Ruminal fermentation and microbial population through supplementation with Clostridium saccharobutylicum. J Microbiol Biotechnol. 2019;29(7):1083-95.

15. Van Immerseel F, Ducatelle R, De Vos M, Boon N, Van De Wiele T, Verbeke $\mathrm{K}$, et al. Butyric acid-producing anaerobic bacteria as a novel probiotic treatment approach for inflammatory bowel disease. J Med Microbiol. 2010; 59(2):141-3.

16. Geirnaert A, Calatayud M, Grootaert C, Laukens D, Devriese S, Smagghe G, et al. Butyrate-producing bacteria supplemented in vitro to Crohn's disease patient microbiota increased butyrate production and enhanced intestinal epithelial barrier integrity. Sci Rep. 2017;7(1):1-14.

17. Novogrodsky A, Dvir A, Ravid A, Shkolnik T, Stenzel KH, Rubin AL, et al. Effect of polar organic compounds on leukemic cells. Butyrate-induced partial remission of acute myelogenous leukemia in a child. Cancer. 1983; 51(1):9-14.

18. Daniel P, Brazier M, Cerutti I, Pieri F, Tardivel I, Desmet G, et al. Pharmacokinetic study of butyric acid administered in vivo as sodium and arginine butyrate salts. Clin Chim Acta. 1989;181(3):255-63. 
19. Macia L, Tan J, Vieira AT, Leach K, Stanley D, Luong S, et al. Metabolitesensing receptors GPR43 and GPR109A facilitate dietary fibre-induced gut homeostasis through regulation of the inflammasome. Nat Commun. 2015; 6:6734.

20. Newmark HL, Lupton JR, Young CW. Butyrate as a differentiating agent: pharmacokinetics, analogues and current status. Cancer Lett. 1994;78(1-3): $1-5$

21. Vinolo MA, Rodrigues HG, Hatanaka E, Sato FT, Sampaio SC, Curi R. Suppressive effect of short-chain fatty acids on production of proinflammatory mediators by neutrophils. J Nutr Biochem. 2011;22(9):84955.

22. Heidor R, Furtado KS, Ortega JF, de Oliveira TF, Tavares PE, Vieira A, et al. The chemopreventive activity of the histone deacetylase inhibitor tributyrin in colon carcinogenesis involves the induction of apoptosis and reduction of DNA damage. Toxicol Appl Pharmacol. 2014;276(2):129-35.

23. Walter J, Mangold M, Tannock GW. Construction, analysis, and $\beta$-glucanase screening of a bacterial artificial chromosome library from the large-bowel microbiota of mice. Appl Environ Microbiol. 2005;71(5):2347-54.

24. Miller DA, Suen G, Bruce D, Copeland A, Cheng J-F, Detter C, et al. Complete genome sequence of the cellulose-degrading bacterium Cellulosilyticum lentocellum. J Bacteriol. 2011;193(9):2357-8.

25. Koeck DE, Maus I, Wibberg D, Winkler A, Zverlov W, Liebl W, et al. Complete genome sequence of Herbinix luporum SD1D, a new cellulose-degrading bacterium isolated from a Thermophilic biogas reactor. Genome Announc 2016;4(4):e00687-16

26. Lagkouvardos I, Pukall R, Abt B, Foesel BU, Meier-Kolthoff JP, Kumar N, et al. The mouse intestinal bacterial collection ( $\mathrm{miBC}$ ) provides host-specific insight into cultured diversity and functional potential of the gut microbiota. Nat Microbiol. 2016;1(10):16131.

27. Fernandez L, Beerthuyzen MM, Brown J, Siezen RJ, Coolbear T, Holland R, et al. Cloning, characterization, controlled overexpression, and inactivation of the major tributyrin esterase gene of Lactococcus lactis. Appl Environ Microbiol. 2000;66(4):1360-8.

28. Tettelin H, Nelson KE, Paulsen IT, Eisen JA, Read TD, Peterson S, et al. Complete genome sequence of a virulent isolate of Streptococcus pneumoniae. Science. 2001;293(5529):498-506

29. Bornscheuer UT. Microbial carboxyl esterases: classification, properties and application in biocatalysis. FEMS Microbiol Rev. 2002;26(1):73-81.

30. Kahya HF, Andrew PW, Yesilkaya H. Deacetylation of sialic acid by esterases potentiates pneumococcal neuraminidase activity for mucin utilization, colonization and virulence. PLoS Pathog. 2017;13(3):e1006263.

31. Yoon MY, Min KB, Lee KM, Yoon Y, Kim Y, Oh YT, et al. A single gene of a commensal microbe affects host susceptibility to enteric infection. Nat Commun. 2016:7:11606.

32. Chen Y, Si J-M, Liu W-L, Cai J-T, Du Q, Wang L-J, et al. Induction of experimental acute ulcerative colitis in rats by administration of dextran sulfate sodium at low concentration followed by intracolonic administration of 30\% ethanol. J Zhejiang Univ Sci B. 2007;8(9):632-7.

33. Chassaing B, Aitken JD, Malleshappa M, Vijay-Kumar M. Dextran sulfate sodium (DSS)-induced colitis in mice. Curr Protoc Immunol. 2014;104:15 25 $1-152514$.

34. Patrascu O, Beguet-Crespel F, Marinelli L, Le Chatelier E, Abraham AL, Leclerc $M$, et al. A fibrolytic potential in the human ileum mucosal microbiota revealed by functional metagenomic. Sci Rep. 2017;7:40248.

35. Yoon MY, Lee KM, Yoon Y, Go J, Park Y, Cho YJ, et al. Functional screening of a metagenomic library reveals operons responsible for enhanced intestinal colonization by gut commensal microbes. Appl Environ Microbiol. 2013;79(12):3829-38

36. Jones BV, Begley M, Hill C, Gahan CG, Marchesi JR. Functional and comparative metagenomic analysis of bile salt hydrolase activity in the human gut microbiome. Proc Natl Acad Sci U S A. 2008;105(36):13580-5.

37. Cheng G, Hu Y, Yin Y, Yang $X$, Xiang C, Wang B, et al. Functional screening of antibiotic resistance genes from human gut microbiota reveals a novel gene fusion. FEMS Microbiol Lett. 2012;336(1):11-6.

38. Tasse L, Bercovici J, Pizzut-Serin S, Robe P, Tap J, Klopp C, et al. Functional metagenomics to mine the human gut microbiome for dietary fiber catabolic enzymes. Genome Res. 2010;20(11):1605-12.

39. de la Cuesta-Zuluaga J, Mueller NT, Alvarez-Quintero R, Velasquez-Mejia EP, Sierra JA, Corrales-Agudelo V, et al. Higher fecal short-chain fatty acid levels are associated with gut microbiome Dysbiosis, obesity, hypertension and Cardiometabolic disease risk factors. Nutrients. 2018;11(1):51.
40. Faradila S, Oetari A, Sjamsuridzal W. Detection of tributyrin utilization by Rhizopus azygosporus UICC 539 at various temperatures. AIP Conf Proc. 2020;2242(1):050003

41. Esteban-Torres M, Mancheno JM, de las Rivas B, Munoz R. Production and characterization of a tributyrin esterase from Lactobacillus plantarum suitable for cheese lipolysis. J Dairy Sci. 2014;97(11):6737-44.

42. Arumugam M, Raes J, Pelletier E, Le Paslier D, Yamada T, Mende DR, et al. Enterotypes of the human gut microbiome. Nature. 2011;473(7346):174-80.

43. Ollis DL, Cheah E, Cygler M, Dijkstra B, Frolow F, Franken SM, et al. The alpha/beta hydrolase fold. Protein Eng. 1992;5(3):197-211.

44. Sheridan PO, Martin JC, Lawley TD, Browne HP, Harris HMB, BernalierDonadille $A$, et al. Polysaccharide utilization loci and nutritional specialization in a dominant group of butyrate-producing human colonic Firmicutes. Microb Genom. 2016;2(2):e000043.

45. Sato FT, Yap YA, Crisma AR, Portovedo M, Murata GM, Hirabara SM, et al. Tributyrin attenuates metabolic and inflammatory changes associated with obesity through a GPR109A-dependent mechanism. Cells. 2020;9(9):2007.

46. Sotira S, Dell'Anno M, Caprarulo V, Hejna M, Pirrone F, Callegari ML, et al. Effects of tributyrin supplementation on growth performance, insulin, blood metabolites and gut microbiota in weaned piglets. Animals. 2020;10(4):726.

47. Wang C, Cao S, Shen Z, Hong Q, Feng J, Peng Y, et al. Effects of dietary tributyrin on intestinal mucosa development, mitochondrial function and AMPK-mTOR pathway in weaned pigs. J Anim Sci Biotechnol. 2019;10:93.

48. Fachi JL, Felipe JDS, Pral LP, da Silva BK, Corrêa RO, de Andrade MCP, et al, Butyrate Protects Mice from Clostridium difficile-Induced Colitis through an HIF-1-Dependent Mechanism. Cell Rep. 2019;27(3):750-61.e7.

49. Seemann T. Prokka: rapid prokaryotic genome annotation. Bioinformatics. 2014;30(14):2068-9.

50. Armenteros JJA, Tsirigos KD, Sønderby CK, Petersen TN, Winther O, Brunak S, et al. SignalP 5.0 improves signal peptide predictions using deep neural networks. Nat Biotechnol. 2019;37(4):420-3.

51. Quinn DM, Shirai K, Jackson RL, Harmony JA. Lipoprotein lipase catalyzed hydrolysis of water-soluble p-nitrophenyl esters. Inhibition by apolipoprotein C-II. Biochemistry. 1982;21(26):6872-9.

\section{Publisher's Note}

Springer Nature remains neutral with regard to jurisdictional claims in published maps and institutional affiliations.
Ready to submit your research? Choose BMC and benefit from:

- fast, convenient online submission

- thorough peer review by experienced researchers in your field

- rapid publication on acceptance

- support for research data, including large and complex data types

- gold Open Access which fosters wider collaboration and increased citations

- maximum visibility for your research: over $100 \mathrm{M}$ website views per year

At $\mathrm{BMC}$, research is always in progress.

Learn more biomedcentral.com/submissions 